\title{
Teaching multilayer optical coatings with coaxial cables
}

\author{
J. Cos, M. M. Sánchez-López, J. Davis, D. Miller, I. \\ Moreno, et al.
}

J. Cos, M. M. Sánchez-López, J. A. Davis, D. Miller, I. Moreno, P. Velásquez, "Teaching multilayer optical coatings with coaxial cables," Proc. SPIE 9664, Ninth International Topical Meeting on Education and Training in Optics and Photonics, 96640Q (24 October 2005); doi: 10.1117/12.2207780

SPIE Event: Ninth International Topical Meeting on Education and Training in Optics and Photonics, 2005, Marseille, France 


\title{
Ref ETOP095
}

Teaching multilayer optical coatings with coaxial cables

\author{
J. Cos ${ }^{1}$, M.M. Sánchez-López ${ }^{1}$, J. A. Davis ${ }^{2}$, D. Miller ${ }^{2}$, I. Moreno ${ }^{3}$ and P. Velásquez ${ }^{3}$
}

${ }^{1}$ Dep. Física y Arquitectura de Computadores, Univ. Miguel Hernández, 03202 Elche, Spain

${ }^{2}$ Department of Physics, San Diego State University, San Diego, California 92182-1233

${ }^{3}$ Dep. Ciencia y Tecnología de Materiales, Universidad Miguel Hernández, 03202 Elche, Spain

\begin{abstract}
We explore the analogies between a system of coaxial cables with periodicity in the impedance, and a system of dielectric stacks with periodicity in the index of refraction. The latter is a photonic crystal with wave propagation control in the optical range, while the former can be regarded as a "coaxial" photonic crystal for radiofrequency control. We reproduce electrical analogs of widely used thin-film optical devices, such as Bragg reflectors, Fabry-Perot resonators and harmonic transmission filters. Coaxial crystals represent an inexpensive way of teaching multilayer optical coatings. We show also that a simple phasor analysis provides an intuitive technique to describe the transmission properties of optical multilayers.
\end{abstract}

\section{Introduction}

\section{Summary}

Optical multilayers are the base of many optical devices. The simplest one consists in alternating layers of materials with high and low refractive index. Although optical multilayers are usually studied in an Optics course in Physics and Engineering degrees [1], it is difficult to perform experiments on this subject in a student laboratory because of the technological complexity in the fabrication of thin-film optical coatings. In this work we show how to use coaxial cables to experiment the physics of wave propagation through optical multilayers. We build a periodically arrangement of cables of high and low impedance, where the impedance plays the role of the refractive index. These structures, known as coaxial photonic crystals [2], reproduce in the radio-frequency range optical multilayer devices such as Bragg reflectors, Fabry-Perot resonators and harmonic transmission filters [3].

\section{Experimental set -up and results}

We have built the electrical analog to the optical multilayer $(H L) N H$, where $H$ and $L$ are the high and low refraction index layers, $N$ times repeated [1]. Each unit cell of the coaxial structure has a $50 \Omega$ cable RG-58/U (low impedance, L) and a $75 \Omega$ cable RG-59/U (high impedance, $\mathrm{H}$ ) connected with barrel connectors. The extreme media are the sweep generator (Wavetek 1062) and the oscilloscope (Tektronix TDS-3054), with $50 \Omega$ ouput and input impedance respectively. To avoid the impedance mismatch at the connection to the generator and the oscilloscope, we connect a $50 \Omega$ cable of arbitrary length at both ends. The transmittance of this coaxial cable structure is obtained theoretically by the transfer matrix method, which is commonly used when studying optical multilayer structures [4].

Figure 1 shows the theoretical and experimental transmitted voltage of the structure $(\mathrm{HL}) \mathrm{NH}$, for $\mathrm{N}=3,10$ and cable lengths of $3 \mathrm{ft}(0.9 \mathrm{~m})$. The theoretical results consider lossless cables. In order to compare them with the measured rms voltage, we have plotted the square root of the calculated transmittance. As we increase the number of unit cells the transmission minimum becomes deeper and wider, eventually yielding a forbidden band in transmission 
that is centered at $55.5 \mathrm{MHz}$. This behaviour is similar to the results with dielectric stacks $[1,4]$. Therefore, the coaxial cable structure $(\mathrm{HL}) \mathrm{NH}$ is the electrical analog of a dielectric mirror or Bragg reflector.

Now, we can build the electrical analog of a Fabry-Perot resonator by connecting a cable (L') between two coaxial mirrors. The structure is: (HL)NH-L'-(HL)NH [3]. Figure 2 shows the transmitted voltage of a coaxial Fabry-Perot with $\mathrm{N}=5$ and L' cable length of $6 \mathrm{ft}$. The theoretical and experimental data agree in the resonance position.
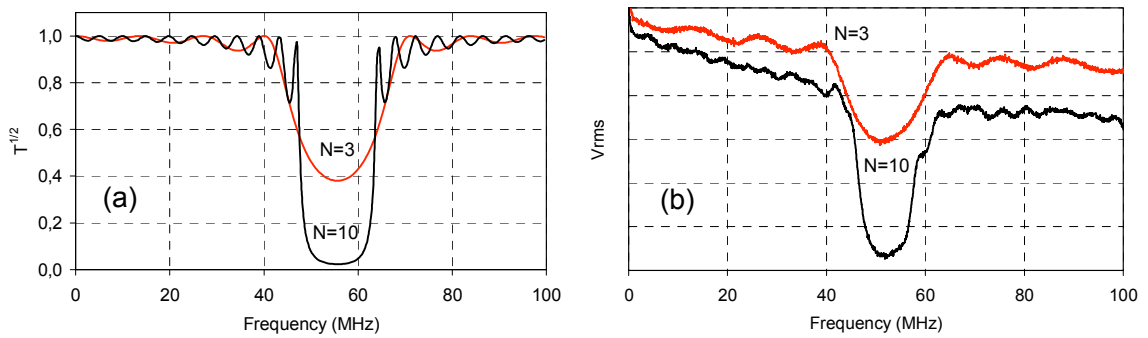

FIGURE 1. Transmitted voltage of the coaxial Bragg reflector $(H L)^{N} H$, with cables $L=(50 \Omega$, $3 \mathrm{ft}), \mathrm{H}=(75 \Omega, 3 \mathrm{ft})$ and $\mathrm{N}=3,10$. (a) Theoretical results, (b) Experimental data.
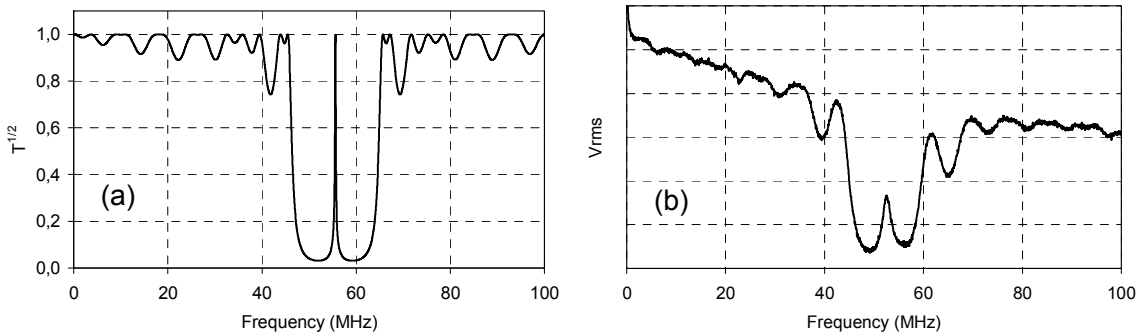

FIGURE 2. Transmitted voltage for a Fabry-Perot $(H L)^{5} H-L^{\prime}-(H L)^{5} H$, with cables $L=(50 \Omega, 3 \mathrm{ft})$ and $H=(75 \Omega, 3 \mathrm{ft})$, and mirror spacing $L^{\prime}=6 \mathrm{ft}$. (a) Theoretical results, (b) Experimental data.

Multilayer harmonic transmission filters can also be mimic with coaxial cables. These filters are Bragg reflectors that transmit different sets of harmonic frequencies for different relative thickness of the $\mathrm{H}$ and $\mathrm{L}$ layers [4]. Figure 3 shows the calculated and measured transmittance for the coaxial structure $(\mathrm{HL})^{4}$, where the $\mathrm{H}$ and $\mathrm{L}$ cable lengths are $\mathrm{D}_{\mathrm{H}}=3 \mathrm{ft}$ and $D_{L}=9 \mathrm{ft}$. There is a gap in transmittance for the first, second and third harmonics, with a fundamental frequency of $27.7 \mathrm{MHz}$. There is no gap at the fourth harmonic. By changing the relative cable lengths $D_{H} / D_{L}$ we can change which harmonics are transmitted [5]. We show that a simple phasor analysis provides an intuitive method for understanding the frequency harmonic aspects of these structures. This phasor analysis is developed from a Fourier transform (FT) technique which is usually applied to the design of inhomogeneous optical coatings [5]. In this FT approach, the transmission properties of non-absorbing multilayer structures are related to the FT of the logarithmic derivative $g(x)$ of the refractive index profile. The FT of $g(x)$ is defined as $\widetilde{Q}(\sigma)=Q(\sigma) \exp (i \phi(\sigma))=\mathrm{FT}[g(x)]$, where $Q(\sigma)$ and $\phi(\sigma)$ are the modulus and the phase of $\widetilde{Q}(\sigma)$, respectively, $\sigma$ is the inverse wavelength. The reflectance is large when $Q$ is large; otherwise the incident beam is transmitted [5].

We apply this FT approach to simple $(\mathrm{HL})^{\mathrm{N}}$ coaxial cable filters like those of Fig. 3, where the refraction index profile $n(x)$ is a rectangular function. The logarithmic derivative $g(x)$ of this function consists in a summation of two sets of delta functions, and its FT can then be written as [5], 


$$
\tilde{Q}(\sigma)=\mathbf{F T}[g(x)]=\frac{1}{d} \sum_{m=-\infty}^{\infty} \tilde{\xi} \cdot \delta\left(\sigma-\frac{m}{d}\right)
$$

where $d$ is the total unit cell double optical thickness and $\xi$ is a complex phasor given by $\widetilde{\xi}=r(1-\exp (-i 2 \pi m a))$, being $r=0.5 \ln \left(Z_{H} / Z_{L}\right)$ and $Z_{H}\left(Z_{L}\right)$ the high (low) cable impedance. The function $\widetilde{Q}(\sigma)$ in Eq. (1) takes maximum values at $\sigma=m / d$. Since $\sigma=1 / \lambda=f / c$, transmittance gaps are expected at the frequencies given by $f_{m}=m v /(2 d)$, where the wave velocity in coaxial cables is $v=2 c / 3$. A multigap transmission spectrum of the $(\mathrm{HL})^{\mathrm{N}}$ structure is expected, where $m$ labels the harmonic frequency. However, some of the transmission gaps will not appear because of the phasor $\xi$. The modulus of $\widetilde{\xi}$ determines the modulus of the function $\widetilde{Q}(\sigma)$, and consequently the depth of the transmission gaps.
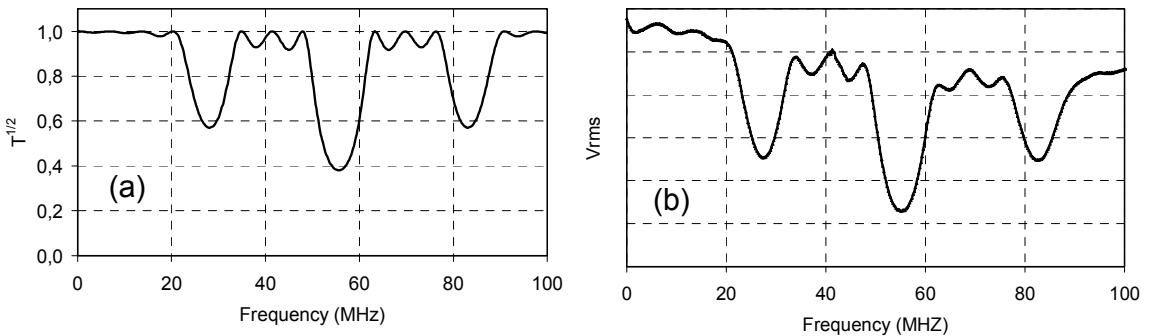

FIGURE 3: Coaxial photonic crystal $(\mathrm{HL})^{4}$ with $\mathrm{H}=\left(75 \Omega\right.$. , $\left.D_{H}=3 \mathrm{ft}\right), \mathrm{L}=\left(50 \Omega, D_{L}=9 \mathrm{ft}\right)$. (a) Calculated transmittance using the exact transfer matrix method. (b). Experimental transmittance.

Considering this theory, we examine the transmission spectra of Fig. 3. Figure 4 shows the phasor diagrams for the two terms of $\tilde{\xi}$ for various harmonic orders $m$. For $m=1,3$ the phasors are in quadrature, thus the first and third transmission minima have equal depths. For $m=2$, the phasors add exactly in phase and the second transmission gap is the deepest. For $m=4$, the two phasors cancel exactly, consequently there is no transmission gap for the fourth harmonic. Other $(\mathrm{HL})^{4}$ structures with different cable length ratio have been similarly analyzed [5], probing the accuracy of this phasor analysis.

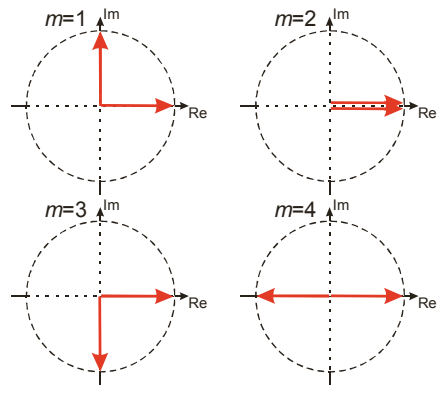

FIGURE 4: Phasor diagrams for the coaxial cable structure of Fig. 3.

\section{Conclusion}

In summary, we have considered a system of coaxial cables with periodicity in the impedance and have built the electrical analogs to multilayer optical devices such as Bragg reflectors, Fabry-Perot resonators and harmonic transmission filters. These simple experiments require equipment that is available in most student laboratories and are a very useful tool to show the physics of multilayer optical coatings. Also, we have shown that a simple phasor diagram derived from a Fourier transform analysis is extremely successful at predicting the frequencies and relative depths of the transmission minima of multilayer optical filters. 


\section{Acknowledgements}

This work was financed by the Spanish Ministerio de Ciencia y Tecnología (BFM200306273-C02-02) and by Generalitat Valenciana (GRUPOS03/117).

\section{References}

1. E. Hecht, Optics, Addison-Wesley (2002), Chap. 9.

2. L. Poirier and A. Haché, Appl. Phys. Lett. 78, 2626-2628 (2001).

3. M.M. Sánchez-López, J. Davis, K. Crabtree, Am.J.Phys. 71 (12), 1314-1319 (2003).

4. H. MacLeod, Thin-film optical filters, Institute of Physics Publishing (1986), Chap. 6.

5. M. M. Sánchez-López, J. Cos, J. A. Davis, D. A. Miller and I. Moreno, Appl. Opt. 44, 37743783 (2005). 